\title{
Interactions between dietary supplements in hospitalized patients
}

\author{
Maurizio Muscaritoli ${ }^{1}$
}

Received: 5 August 2016/Accepted: 9 August 2016/Published online: 20 August 2016

(C) SIMI 2016

In this issue of the Journal, Levy et al. [1] report on the clinically relevant results of a cross-sectional prospective study, performed by a multidisciplinary team of researchers between 2009 and 2014, and aimed at detecting dietary and herbal supplements (DHSs) use among patients hospitalized at the Bnai Zion Medical Center in Israel.

The innovative aspect of Levy's study was represented by the attempt to identify through the administration of a dedicated questionnaire, not only the prevalence of DHS use among hospital patients, but also the possible DHSDHS interactions in the inpatient study population. Indeed, the hypothesis of the authors is that DHS-DHS interaction may be a cause for either hospitalization or for worsening of an underlying comorbidity. The rate of respondents to the questionnaire was rather good, reaching almost $91 \%$ (927 out of the 1020 delivered questionnaires). Interestingly enough, almost $50 \%$ of the respondents overall reported the use of some 89 different DHSs. The potential risks for DHS-DHS interactions were analyzed through univariate and multivariate logistics regression analyses.

In their study, Levy et al. identified potential DHS-DHS interactions in only 59 patients, accounting for $13 \%$ of respondents, with the most common potential effects being hypercalcemia, hypotension, bleeding, and hypoglycemia. In three patients, these negative interactions were associated with either hospitalization or adverse event during hospital stay. With respect to their working hypothesis, the authors then conclude that more than $6 \%$ of patients

Maurizio Muscaritoli

maurizio.muscaritoli@uniroma1.it

1 Department of Clinical Medicine, Sapienza University of Rome, Viale dell'Università, 37, 00185 Rome, Italy hospitalized in a general hospital have the potential for developing DHS-DHS interaction.

Although the study has limitations, some of which are acknowledged by the author themselves in the discussion, the report by Levy et al. raises a relevant issue in the modern scenario of clinical medicine, i.e., the rising consumption of dietary supplements by patients in the community, as a key component of complementary and alternative medicines (CAMs) [2].

The use of CAM such as herbal medicines and dietary supplements is well documented, and is particularly common in older adults [3] and in patients with chronic diseases including cancer [2]. Just to give an estimate of the phenomenon, it can be considered that the number of dietary supplements in the USA grew from 4000 in 1994 to approximately 75,000 in 2008 [4], for many of which manufacturers evade regulatory limits [5].

The growing use of herbal and dietary supplements is favored by the ease of internet-acquired information and internet trading, coupled with the diffuse perception that while conventional medicines are often misprescribed, overprescribed, ineffective or dangerous [6], and herbal and dietary remedies represent a natural and, therefore, safer therapeutic approach to maladies, especially chronic and incurable diseases. For these reasons, as correctly pointed out by Levy et al. [1], their use is very commonly not known by doctors $[7,8]$. Indeed, Levy et al. report that in their study, DHS use was not reported in $89 \%$ of the medical files of the studied patients, and that only $18 \%$ of the DHSs involved in the interactions were documented.

These findings highlight two important aspects that should not be overlooked in our everyday clinical practice. On the one side, is the reluctance of patients to advise their physician about the regular use of DHS, since this information is deemed not important for the doctor. On the other 
side, is the need to train medical students and residents to systematically and accurately include information about the use of dietary supplements in the pharmacological history of in- and outpatients they are taking care of. The latter appears particularly relevant when considering the multiethnic scenario of modern clinical medicine [9] and the culture-based wide use of herbal remedies in nonEuropean, and particularly in Asian populations [10]. This represents a real challenge clinical medicine will have to undertake in the next few years.

A category of patients who appear vulnerable and exposed to the risks of health- or life-threatening interactions of dietary supplements is represented by cancer patients, who are particularly keen to refer to CAM worldwide, [2] and to take herbal medicines and dietary supplements in the attempt to attenuate or counteract the side effects of conventional antineoplastic treatments, or even in the hope of enhancing their therapeutic efficacy $[5,11]$.

Amazingly enough, almost $60 \%$ of people consuming dietary supplements do so to prevent or treat cancer [5], which explains why Americans spent more than 36 billion USD on dietary supplements in 2014 [5]. In the United States, almost half of cancer patients start taking dietary supplements soon after receiving a diagnosis of cancer [12]. Although solid clinical data are lacking [2], concern still exists about the potential interactions of dietary supplements with conventional therapies (either radiochemo- or biological therapies) especially with chemotherapy drugs with a narrow therapeutic index, [2] or with therapies based on the induction of reactive oxygen species [13].

In conclusion, the conventional clinical medicine is progressively facing the growing diffusion of CAM, with treatments based on the claimed efficacy of DHS to exert positive effects in patients with acute and chronic diseases, including cancer. Therapeutic or preventative claims for most DHS are based on evidence from phase 2 trials, which in the majority of cases fail to be confirmed in phase 3 clinical trials [5]. However, DHSs fuel a very florid market, since patients often base significant expectations on these treatments, reporting subjective improvement in disease- or treatment-related symptoms. This has clinical and ethical implications that should be carefully evaluated by well-trained, modern physicians. Modes of actions, possible biologically plausible benefits, possible DHD-DHS and DHS-drug interactions and patient's expectations should all be taken into account when suggesting a patient to continue or withdraw a
DHS-based treatment. Provided that we have taken note of it in the medical history.

\section{Compliance with ethical standards}

Conflict of interest The Author declares that he has no conflict of interest.

Statements on human and animal rights This article does not contain any studies with human participants or animals performed by any of the authors.

Informed consent None.

\section{References}

1. Levy I, Attias S, Ben Arye E, Goldstein L, Schiff E (2016) Interactions between dietary supplements in hospitalized patients. Intern Emerg Med. doi:10.1007/s11739-015-1385-3

2. Alsanad SM, Williamson EM, Howard RL (2014) Cancer patients at risk of herb/food supplement-drug interactions: a systematic review. Phytother Res 28:1749-1755

3. Pitkälä KH, Suominen MH, Bell JS, Strandberg TE (2016) Herbal medications and other dietary supplements. A clinical review for physicians caring for older people. Ann Med 16:1-17 (Epub ahead of print)

4. US Government Accountability Office. Dietary supplements: FDA should take further actions to improve oversight and consumer understanding. Published January 29, 2009. http://www. gao.gov/products/GAO-09-250. Accessed 30 July 2016

5. Paller CJ, Denmeade SR, Carducci MA (2016) Challenges of conducting clinical trials of natural products to combat cancer. Clin Adv Hematol Oncol 14:447-455

6. Zaffani S, Cuzzolin L, Benoni G (2006) Herbal products: behaviors and beliefs among Italian women. Pharmacoepidemiol Drug Saf 15:354-359

7. Eisenberg DM, Davis RB, Ettner SL et al (1998) Trends in alternative medicine use in the USA, 1990-1997; results of a follow-up national survey. JAMA 280:1569-1575

8. Eisenberg DM, Kessler RC, Van Rompay MI et al (2001) Perceptions about complementary therapies relative to conventional therapies among adults who use both: results from a national survey. Ann Intern Med 135:344-351

9. Barata AN, Rigon S (2015) Family medicine $360^{\circ}$ : global exchanges in family medicine. J Fam Med Prim Care 4:305-309

10. Liu T, Li X, Zou ZY, Li C (2015) The prevalence and determinants of using traditional Chinese medicine among middle-aged and older Chinese adults: results from the China health and retirement longitudinal study. J Am Med Dir Assoc 16:1002.e1-5

11. Gröber U, Holzhauer P, Kisters K, Holick MF, Adamietz IA (2016) Micronutrients in oncological intervention. Nutrients 12(8):163

12. Patterson RE, Neuhouser ML, Hedderson MM, Schwartz SM, Standish LJ, Bowen DJ (2003) Changes in diet, physical activity, and supplement use among adults diagnosed with cancer. J Am Diet Assoc 103(3):323-328

13. Yasueda A, Urushima H, Ito T (2016) Efficacy and interaction of antioxidant supplements as adjuvant therapy in cancer treatment: a systematic review. Integr Cancer Ther 15:17-39 